\title{
Anna Moskal, Im Spannungsfeld von Region und Nation. Die Polonisierung der Stadt Posen nach 1918 und 1945
}

Wiesbaden : Harrassowitz Verlag (Studien zur Sozial- und Wirtschaftsgeschichte Ostmitteleuropas, 23), 2013, 298p., $56 €$.

\section{Thomas Serrier}

\section{OpenEdition}

\section{Journals}

Édition électronique

URL : http://journals.openedition.org/ifha/8407

DOI : 10.4000/ifha.8407

ISSN : 2198-8943

Éditeur

IFRA - Institut franco-allemand (sciences historiques et sociales)

Référence électronique

Thomas Serrier, «Anna Moskal, Im Spannungsfeld von Region und Nation. Die Polonisierung der Stadt Posen nach 1918 und 1945 », Revue de l'IFHA [En ligne], Date de recension, mis en ligne le 01 février 2016, consulté le 22 septembre 2020. URL : http://journals.openedition.org/ifha/8407 ; DOI : https:// doi.org/10.4000/ifha.8407

Ce document a été généré automatiquement le 22 septembre 2020.

(CIFHA 


\title{
Anna Moskal, Im Spannungsfeld von Region und Nation. Die Polonisierung der Stadt Posen nach 1918 und 1945
}

\author{
Wiesbaden : Harrassowitz Verlag (Studien zur Sozial- und \\ Wirtschaftsgeschichte Ostmitteleuropas, 23), 2013, 298p., $56 €$.
}

\section{Thomas Serrier}

Dans un texte resté fameux, "Depozyt », le dissident polonais Jan-Jozef Lipski avait plaidé en plein tournant des années 1989-1990 pour que les Polonais prennent enfin conscience des enjeux non pas simplement conservatoires, mais bien politiques, voire éthiques, du patrimoine culturel et architectural allemand dont la Pologne était devenue le « dépositaire » à l'ouest après la vaste translation des frontières de 1945. La conscience d'un "patrimoine partagé ", pour reprendre les termes de l'historien de l'art Andrzej Tomaszewski, s'est aujourd'hui largement diffusée, au point de constituer du milieu des années 1990 à la fin des année 2000 un véritable „main stream“ de la recherche en histoire et en histoire de l'art de l'Europe centrale et orientale, particulièrement spectaculaire dans le cas germano-polonais. Portés par le paradigme déconstructiviste, nombre d'ouvrages ont alors concentré leur attention sur la "construction“ du national, les processus multiples et pluriels d'appropriation culturelle et les politiques successives de nationalisation du patrimoine marqué au sceau de l'Altérité. L'ouvrage d'Anna Moskal, consacré à la « polonisation de la ville de Posen après 1918 et 1945 ", comme l'indique son sous-titre, s'inscrit donc dans un champ déjà largement balisé par les ouvrages de Gregor Thum ou Norman Davies (sur Breslau/Wrocław), de Peter Oliver Loew (Danzig/Gdańsk), de Jan Musekamp (Stettin/ Szczecin), auxquels il convient d'ajouter les études sur d'autres espaces (Per Brodersen sur Königesberg/Kaliningrad, Felix Ackermann sur Grodno, Christoph Mick sur Lemberg/Lwóv, etc.) ou d'autres époques - comme cette fin du XIXe siècle, marquée, dans la Posnanie sous administration prussienne, par une politique de germanisation toujours plus étendue et englobant le patrimoine, réinterprété ou créé de toutes pièces (Thomas Serrier, Stefan Dyroff). 
Issue d'une thèse doctorale dirigée par Philipp Ther, la belle étude d'Anna Moskal parvient à enrichir ce domaine de la recherche de nombreux apports originaux. La ville étudiée est justement la capitale successive de la Wielkopolska (Grande Pologne) et de la Posnanie, Posen/Poznań, dont l'histoire locale a été marquée par la concurrence des nationalismes allemand et polonais aux $\mathrm{XIX}^{\mathrm{e}}$ et $\mathrm{XX}^{\mathrm{e}}$ siècles. Polonais et Allemands revendiquaient chacun la suprématie sur la ville, qui changea plusieurs fois de souveraineté: en 1793, 1918, 1939 et 1945. Fallait-il, comme Anna Moskal (p. 5), parler de " polonisation » ou plutôt de " repolonisation » - ou éviter ce terme trop politique, trop peu scientifique et surtout trop limpide pour décrire des situations toujours complexes, ambivalentes et changeantes ? Là n'est pas l'essentiel. L'auteure dresse de fait un tableau nuancé des processus d'appropriation culturelle de l'héritage culturel allemand par la population polonaise, en montrant combien le rapport à un environnement urbain pluriculturel fut avant tout conditionné par la présence ou non des populations allogènes. La concomitance des migrations forcées des Allemands, soumis à l'option d'adopter la citoyenneté polonaise après 1918, ce que ne fit qu'une infime partie d'entre eux, et objets des expulsions ou «transferts» de population entérinés à Potsdam en 1945, fournit le cadre politique et démographique des trois coups de sonde que propose Anna Moskal. Trois études empiriques principales constituent en effet la charpente de cette étude : la transmutation de l'opéra de Posen en scène nationale polonaise, les destinées de la Foire internationale de Posen transformée en haut-lieu de la vie économique nationale polonaise; enfin le sort réservé aux cimetières des trois principales confessions représentées dans l'histoire de la ville, les cimetières catholiques, protestants et juifs. La diversité même des cas approfondis aboutit à un panorama à la fois vaste et différencié.

La « polonisation » est particulièrement lisible autour du cas de la Foire internationale de Posen. Sa continuation à Poznań après 1918 peut se lire comme une tentative de réplique nationale à la grande Exposition de l'Allemagne orientale organisée du temps de la Prusse en 1911, dont l'objectif explicite avait été de faire la démonstration de la supériorité de la civilisation allemande en matière de techniques et d'industrie. Moskal aurait d'ailleurs pu ajouter la mémoire de la grande foire-exposition organisée en 1913 dans un semblable esprit national dans la métropole voisine de Breslau. Les foiresexpositions organisées à Poznań dans la Pologne retournée à l'indépendance de l'entredeux-guerres montrent en creux la permanence d'un complexe allemand. Elles illustrent aussi l'importance de Poznań dans les cartes mentales des Polonais, la capitale de la Wielkopolska étant littéralement surdéterminée par sa fonction symbolique anti-allemande. Le salon de l'agriculture de Poznań de 1929 est visiblement chargé de montrer la „polonité" indubitable de la région à ses visiteurs.

Avec la transformation du théâtre allemand de Posen en opéra national de Poznan, l'auteure aborde l'un des monuments architecturaux les plus symboliques du „forum impérial“ de Posen, cet espace érigé de toutes pièces autour du "château impérial » (1910) dans le cadre de la politique de "relèvement culturel de la Marche orientale » voulue par l'Empereur Guillaume II au cours de la décennie 1898-1914. Le conflit national avant 1914 ayant déjà vu la naissance et la construction d'un théâtre polonais en 1875, les acteurs de la vie culturelle polonaise à Poznań disposaient désormais de deux lieux prestigieux. La nationalisation du théâtre après 1918 engloba autant des modifications symboliques de l'ensemble architectural dans sa matérialité de pierre et ses connotations germaniques que l'introduction massive du répertoire national polonais. La dernière étude dédiée au sort des cimetières après le départ des Allemands 
montre que les projets de transformer tout ou partie de cimetières, notamment ceux des communautés protestantes, en parcs municipaux, phénomène fréquent après 1945 dans les territoires polonais dits "recouvrés ", anciennement allemands, avaient déjà été discutés dans l'entre-deux-guerres. Malgré certains déséquilibres de traitement, l'un des principaux intérêts de l'analyse tient ainsi à la comparaison de deux époques, à travers la " polonisation » de 1918 et celle de 1945, qui met comme ici en lumière, les antécédents, les reprises ou les bifurcations d'un après-guerre à l'autre. Un autre enseignement découle de l'intéressante contextualisation spatiale qui replace les développements politiques et culturels de Poznań dans la dialectique entretenue par la capitale de la Wielkopolska, et, au-delà, des territoires occidentaux de la Pologne, avec la capitale nationale. Fidèle à l'ambition de son titre, centré sur la tension «entre région et nation ", l'ouvrage n'apporte donc pas seulement une nouvelle pierre à l'étude de l'histoire des imbrications germano-polonaises, mais aussi une réflexion appuyée sur une recherche empirique dense et représentative autour de la thématique classique du centre et de la périphérie.

Vous trouverez la table des matières ici : http://d-nb.info/1026253861/04.

INDEX

Index chronologique : Époque contemporaine

Thèmes : Histoire des États et des pouvoirs, Histoire des villes et des régions, Histoire sociale, Histoire de la culture

\section{AUTEUR}

THOMAS SERRIER

Université Paris8/Europa-Universität Viadrina Frankfurt(Oder) 